\title{
Uterine Arteriovenous Malformation Acquired after Cesarean Section: A Case Report
}

\author{
Ana Lúcia Isotton 1,2, Leandro Armani Scaffaro', Ellen Machado Arlindo1,3, Anne Bergmann1,3, \\ Nicolas Karpouzas Vicentini², Janete Vettorazzi ${ }^{1,3^{*}}$
}

${ }^{1}$ Service of Obstetrics and Gynecology and Interventional Radiology Department, Hospital Moinhos de Vento, Porto Alegre, Porto Alegre/RS, Brazil

${ }^{2}$ Medicina Fetal Porto Alegre, Porto Alegre/RS, Brazil

${ }^{3}$ Post Graduation Program in Health Sciences: Gynecology and Obstetrics, Faculdade de Medicina da Universidade Federal do Rio Grande do Sul (UFRGS), RS, Brazil

Email: *jvettorazzi@hcpa.edu.br

How to cite this paper: Isotton, A.L., Scaffaro, L.A., Arlindo, E.M., Bergmann, A., Vicentini, N.K. and Vettorazzi, J. (2021) Uterine Arteriovenous Malformation Acquired after Cesarean Section: A Case Report. Open Journal of Obstetrics and Gynecology, 11, 1512-1516.

https://doi.org/10.4236/ojog.2021.1111142

Received: October 4, 2021

Accepted: November 15, 2021

Published: November 18, 2021

Copyright $\odot 2021$ by author(s) and Scientific Research Publishing Inc. This work is licensed under the Creative Commons Attribution International License (CC BY 4.0).

http://creativecommons.org/licenses/by/4.0/

\begin{abstract}
Background: Uterine arteriovenous malformation (AVM) is a rare, potentially life-threatening cause of abnormal uterine bleeding that can be acquired following a cesarean section. The diagnosis is difficult, often misdiagnosed with retained products of conception and placenta accreta. Transarterial embolization (TAE) is an efficacious and effective method of treating AVM, particularly in patients of reproductive age. Case Report: A 41-year-old, nulliparous woman, started with a history of abnormal uterine bleeding 30 days after a cesarean section. She didn't have anaemia and beta-human chorionic gonadotropin (beta-hCG) was negative. The transvaginal color Doppler ultrasound showed multiple vascular channels within myometrium showing colored mosaic patterns suggesting AVM, confirmed by Magnetic Resonance Imaging. The patient was referred to perform a Computed Tomography Angiography and right uterine artery embolization without any vascular complications. Conclusion: AVM is a rare consequence of cesarean section but has to be considered in cases of persistent uterine bleeding in the puerperium. Embolization is a safe and effective choice treatment of AVM and an alternative and less invasive option for patients wishing to preserve fertility.
\end{abstract}

\section{Keywords}

Arteriovenous Malformation, Embolization Therapeutic, Vaginal Bleeding, Cesarean Section

\section{Introduction}

Acquired uterine arteriovenous malformation (AVM) is a rare condition after a 
cesarean section [1]. This condition is predominantly related to various forms of iatrogenic trauma such as hysteroscopy, curettage and myomectomy; even normal vaginal delivery could be traumatic enough to cause AVM. Endometrial carcinoma, cervical carcinoma and gestational trophoblastic disease could be other causes of AVM [1] [2]. The incidence and the prevalence are difficult to determine because it's often misdiagnosed with retained products of conception and placenta accreta [3]. AVM represents $1 \%$ to $2 \%$ of all genital hemorrhages [3]. The affected patients may have a wide spectrum of clinical signs, such as menorrhagia and menometrorrhagia, lower abdominal pain, dyspareunia and anaemia. Some cases may be asymptomatic [4]. The choice of treatment varies from hormonal treatment (estrogen and progesterone, oral contraceptive, and intrauterine contraceptive devices), open surgical or Transarterial Embolization (TAE). In the past, treatment for symptomatic acquired uterine AVM required hysterectomy [1]. However, endovascular treatment has provided an alternative and less invasive treatment option for patients wishing to preserve fertility [5]. In this report, we present a case of AVM that was successfully treated by TAE.

\section{Case}

A 41-year-old caucasian woman, primiparous, in vitro fertilization pregnancy, came with an intermittent vaginal bleeding started 30 days after cesarean section due to premature rupture of membranes, unfavorable uterine cervix and no desire for induction. Operative reports from the cesarean section showed an intact placenta and an empty uterus. The patient didn't use any contraception and didn't have anaemia. The beta-human chorionic gonadotropin (beta-hCG) was negative. The transvaginal color Doppler ultrasound showed multiple vascular channels within myometrium showing colored mosaic patterns suggesting AVM (Figure 1). These findings were confirmed by Magnetic Resonance Imaging (Figure 2(A) and Figure 2(B)). The patient was then referred to the interventional radiology department to perform a Computed Tomography Angiography and uterine artery embolization. After femoral approach and superselective microcatheterization of uterine arteries, an abnormal vascular pattern was found in

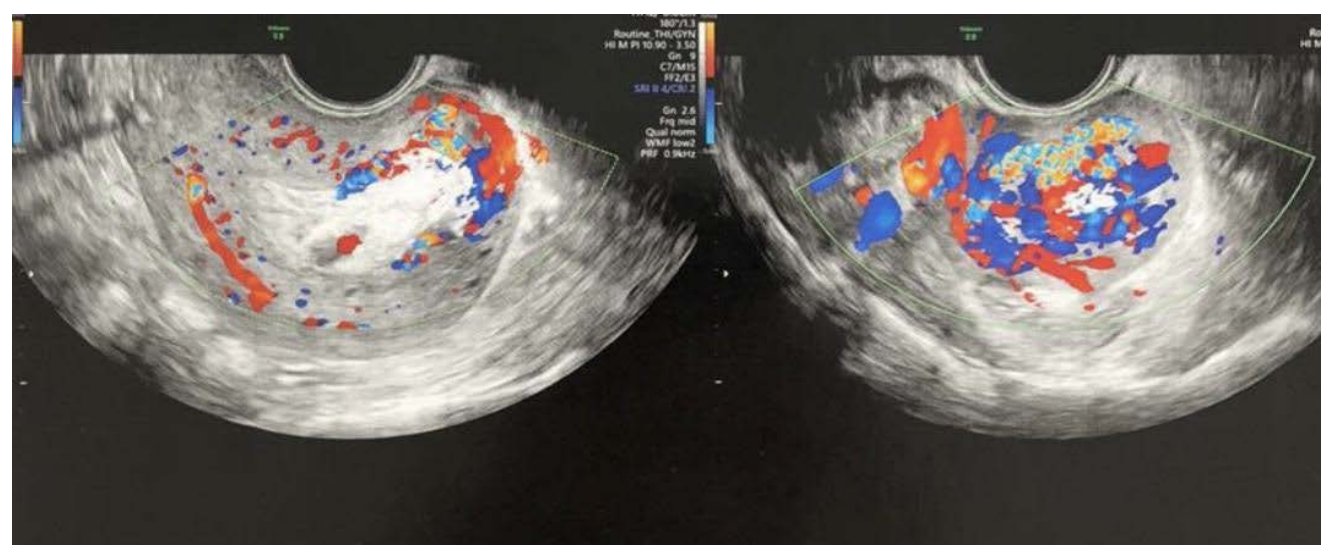

Figure 1. Transvaginal color Doppler ultrasound image showing AVM. 

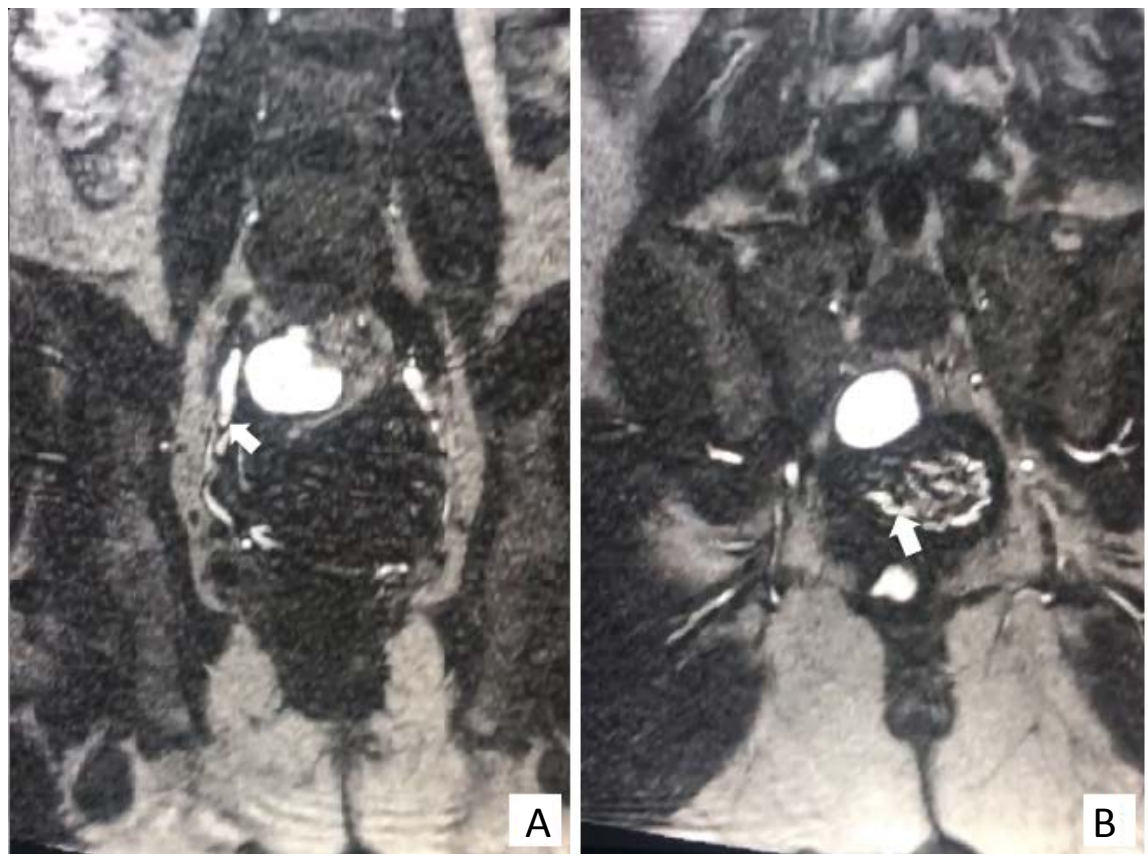

Figure 2. (A) Magnetic Ressonance Imaging showing right uterine artery (arrow); (B) right uterine artery and communication with anomalous vessels (arrow).

the right uterine artery, with distal angiodysplasia and arteriovenous fistula. Uterine artery embolization (UAE) was performed under moderate sedation using the right transfemoral approach according to the Seldinger technique in an angiographic set. First, a contra-lateral approach of left common and internal iliac artery was accessed using 5 French (F) Roberts Uterine Catheter (Cook, Bloomington, IN, USA). Selective angiography did not show abnormal uterine vascularization (Figure 3(A)). Same features were found after left superselective uterine artery catheterization using $2.8 \mathrm{~F}$ microcatheter (Progreat, Terumo). After angiographic confirmation of normal left uterine supply, ipsilateral approach was performed with the same $5 \mathrm{~F}$ catheter. Selective Right internal iliac artery confirmed arteriovenous fistula in distal branches of uterine artery. Superselective uterine artery catheterization was done using $2.8 \mathrm{~F}$ microcatheter (Progreat, Terumo). Selective embolization was performed under fluoroscopic guidance into distal right uterine artery (Figure 3(B) and Figure 3(C)). At first we choose nonspherical polyvinyl alcohol 700 micras (PVA) particles since the fistula debit seemed to be very low. Satisfactory fistula flow occlusion was shown in angiographic controls after PVA embolization, precluding use of coils or N-butyl-2-cyanoacrylate (NBCA, Histoacryl, Braun, Germany). Angiography after embolization showed satisfactory occlusion of these abnormal vessels, without any vascular complications (Figure 3(D)). She had a history of a small vaginal bleeding 10 days after the procedure. Forty days post-embolization the patient was submitted to a control transvaginal ultrasound which showed no abnormal results. Pelvis Magnetic Resonance Imaging was then performed 2 months after the embolization and showed no abnormalities. 


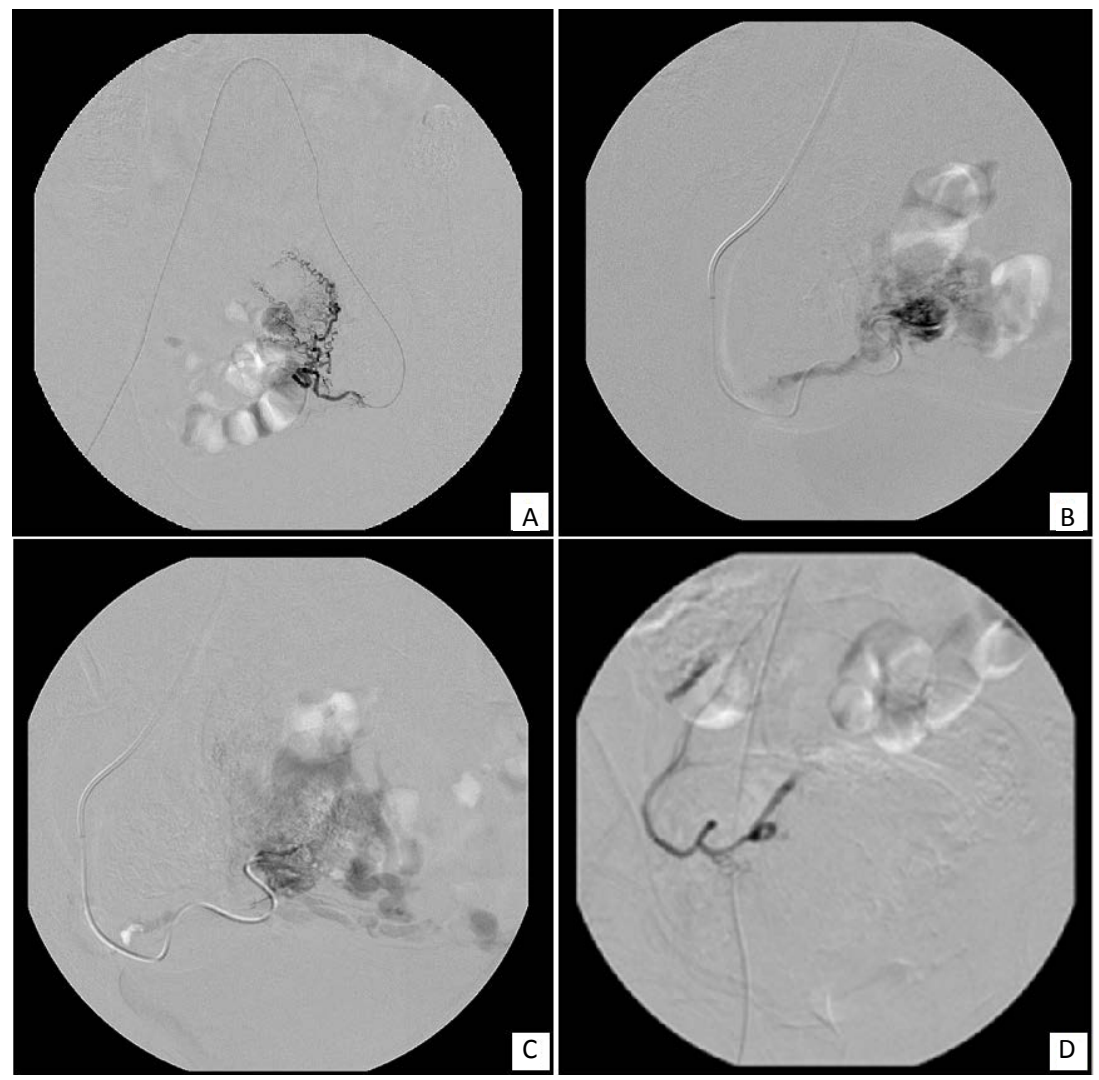

Figure 3. (A) Normal pattern of left uterine artery; (B and C) Superselective contrast injection in the right uterine artery showing arteriovenous fistula with ectasic drainage veins; (D) Right uterine artery after embolization with fistula occlusion.

\section{Discussion}

AVM is a rare consequence of cesarean section and is related to uterine artery trauma during the procedure [6].

The diagnosis is difficult but has to be considered in patients with a history of cesarean section [7].

AVM must be part of the differential diagnosis as a possible cause of persistent bleeding in the puerperium, as well as placenta accreta. MRI is essential for differential diagnosis [1].

The patient usually comes with postpartum bleeding after a cesarean procedure and could cause hypovolemic shock. Sometimes, the patient needs transfusion and hospitalization [8].

In the past, treatment for symptomatic acquired uterine AVM required hysterectomy [1]. However, endovascular treatment has provided an alternative and less invasive treatment option for patients wishing to preserve fertility [1] [5].

Endovascular techniques are often safe and effective in bleeding control, through selective embolization [1].

\section{Conclusion}

AVM could be related to the increased rates of cesarean delivery. As mentioned 
previously, the diagnosis is hard but must be considered in a patient with history of cesarean section. If possible, Transarterial Embolization should be the first choice of treatment. Hysterectomy could be an alternative in those patients that can't perform TAE.

\section{Conflicts of Interest}

The authors declare no conflicts of interest regarding the publication of this paper.

\section{References}

[1] Harzif, A.K., Haloho, A., Silvia, M., et al. (2019) Trans-Arterial Embolization of Acquired Uterine Arteriovenous Malformation after Cesarean Section: A Case Series. International Journal of Reproductive BioMedicine, 17, 135-142. https://doi.org/10.18502/ijrm.v17i2.3991

[2] Szpera-Gozdziewicz, A., Gruca-Stryjak, K., Breborowicz, G.H., et al. (2018) Uterine Arteriovenous Malformation: Diagnosis and Management. Ginekologia Polska, 89, 276-279. https://doi.org/10.5603/GP.a2018.0047

[3] Farias, M.S., Santi, C.C., Lima, A.A.A., et al. (2014) Radiological Findings of Uterine Arteriovenous Malformation: A Case Report of Unusual and Life-Threatening Cause of Abnormal Vaginal Bleeding. Radiologia Brasileira, 47, 122-124. https://doi.org/10.1590/S0100-39842014000200016

[4] Timor-Tritsch, I.E., Haynes, M.C., Monteagudo, A., et al. (2016) Ultrasound Diagnosis and Management of Acquired Uterine Enhanced Myometrial Vascularity/Arteriovenous Malformations. American Journal of Obstetrics and Gynecology, 214, 731.e1-731.e10. https://doi.org/10.1016/j.ajog.2015.12.024

[5] Yoon, D.J., Jones, M., Al Taani, J., Buhimschi, C., et al. (2016) A Systematic Review of Acquired Uterine Arteriovenous Malformations: Pathophysiology, Diagnosis, and Transcatheter Treatment. American Journal of Perinatology Reports, 6, e6-e14. https://doi.org/10.1055/s-0035-1563721

[6] Khan, S., Saud, S., Khan, I., et al. (2019) Acquired Uterine Arteriovenous Malformation Following Dilatation and Curettage Treated with Bilateral Uterine Artery Embolization: A Case Report. Cureus, 11, e4250. https://doi.org/10.7759/cureus.4250

[7] Scribner, D. and Fraser, R. (2016) Diagnosis of Acquired Uterine Arteriovenous Malformation by Doppler Ultrasound. Journal of Emergency Medicine, 51, 168-171. https://doi.org/10.1016/j.jemermed.2016.04.028

[8] Tan, C.C.P., Mathur, M. and Lo, R.H.G. (2017) Uterine Arteriovenous Malformation: A Rare Cause of Secondary Postpartum Hemorrhage. Journal of Medical Cases, 8, 152-154. https://doi.org/10.14740/jmc2804w 